31 Matsumoto H, Niimi A, Jinnai M, et al. Association of alveolar nitric oxide levels with pulmonary function and its reversibility in stable asthma. Respiration 2011; 81: 311-317.

32 Nihlberg K, Andersson-Sjöland A, Tufvesson E, et al. Altered matrix production in the distal airways of individuals with asthma. Thorax 2010; 65: 670-676.

33 Van Veen IH, Sterk PJ, Schot R, et al. Alveolar nitric oxide versus measures of peripheral airway dysfunction in severe asthma. Eur Respir J 2006; 27: 951-956.
34 Verbanck S, Schuermans D, Vincken W. Inflammation and airway function in the lung periphery of patients with stable asthma. J Allergy Clin Immunol 2010; 125: 611-616.

35 Williamson PA, Clearie K, Menzies D, et al. Assessment of smallairways disease using alveolar nitric oxide and impulse oscillometry in asthma and COPD. Lung 2011; 189: 121-129.

\title{
Efficacy of nebulised liposomal amphotericin B in the attack and maintenance treatment of ABPA
}

\section{To the Editors:}

Allergic bronchopulmonary aspergillosis (ABPA) is a pulmonary disorder that results from a hypersensitivity reaction to Aspergillus spp. It has been estimated to occur in 1-3\% of people with chronic asthma and $2-15 \%$ of those with cystic fibrosis [1]. The natural history of ABPA is characterised by exacerbations that can threaten the patient's survival and prognosis [1]. Repetition of such exacerbations is responsible for the development of bronchiectasis, permanent obstructive ventilation defect or fibrotic lung lesions. Prognosis mainly depends on the very early treatment of exacerbations before bronchiectasis sets in. In 2008, the guidelines of the Infectious Diseases Society of America advised combination therapy in ABPA [2]: systemic glucocorticoids to limit the inflammatory component and antifungals to limit mycelium proliferation. However, even though glucocorticoid therapy and antifungals are the treatment of choice for acutestage ABPA and exacerbations, there are no data to guide the duration of this treatment. Therefore, the current objectives for the management of ABPA are a decrease in the frequency and duration of exacerbations, and a limited solicitation of glucocorticoids. Consequently, the maintenance treatment in the management of ABPA remains a current and progressive problem for pneumologists.

We describe a case of ABPA that was difficult to control using the standard treatment regimen, but which improved dramatically and durably following administration of nebulised liposomal amphotericin B (LAmB).

In May 2010, a 67-yr-old female presented with fever and productive cough, with sputum plugs and a history of epilepsy treated with phenobarbital. In the preceding 3 months, she had presented recurrent chest infections resistant to amoxicillin and ciprofloxacin; glucocorticoids $\left(1 \mathrm{mg} \cdot \mathrm{kg}^{-1} \cdot \mathrm{day}^{-1}\right.$ prednisolone for 2 weeks) had been started without any clear improvement.

At that time, ABPA was suspected. A thoracic computed tomography (CT) scan showed right upper lobe consolidation with a bronchocele and the patient's absolute eosinophil count was 1,170 cells $\cdot \mu \mathrm{L}^{-1}$. Further investigations showed increased total immunoglobulin $(\mathrm{Ig}) \mathrm{E}\left(1,154 \mathrm{IU} \cdot \mathrm{mL}^{-1}\right)$ and specific antiAspergillus fumigatus IgE levels $\left(12.9 \mathrm{kU} \cdot \mathrm{L}^{-1}\right)$, and the presence of specific anti- $A$. fumigatus precipitins in the serum (four lines).
Corticosteroids were maintained for 2 weeks $\left(1 \mathrm{mg} \cdot \mathrm{kg}^{-1} \cdot \mathrm{day}^{-1}\right)$ after which the dose was reduced (5-mg decrease every 2 weeks) and itraconazole was added (200 $\mathrm{mg}$ b.i.d.).

After 4 weeks of treatment, the patient's clinical status deteriorated and new consolidations appeared on the CT scan (fig. 1a and $b$ ); due to phenobarbital interaction, plasma itraconazole concentration was very low, so itraconazole treatment was withdrawn. The patient was therefore treated with the following regimen: prednisolone $\left(0.5 \mathrm{mg} \cdot \mathrm{kg}^{-1} \cdot \mathrm{day}^{-1}\right.$ for 2 weeks, then reduced by 5 mg every 2 weeks until discontinuation), associated with nebulisations of LAmB ( $25 \mathrm{mg}$ twice weekly) until steroids were stopped, followed by a maintenance dose of LAmB (25 mg once weekly) to prevent subsequent ABPA exacerbations. As shown on figure $1 \mathrm{c}$ and $\mathrm{d}$, the patient improved dramatically and durably, with a significant decrease in eosinophil count, precipitins and total and specific IgE levels over time (values after 6 months of LAmB were 370 cells $\mu \mathrm{L}^{-1}$, two lines, $133 \mathrm{IU} \cdot \mathrm{mL}^{-1}$ and $3.05 \mathrm{kU} \cdot \mathrm{L}^{-1}$, respectively, and 6 months after discontinuation of $\mathrm{LAmB}$ were 310 cells $\cdot \mu \mathrm{L}^{-1}$, two lines, $236 \mathrm{IU} \cdot \mathrm{mL}^{-1}$ and $3.28 \mathrm{kU} \cdot \mathrm{L}^{-1}$, respectively). After 2 months of this regimen, prednisolone was stopped and nebulised LAmB continued ( $25 \mathrm{mg}$ once weekly) for 6 months as maintenance therapy. In our case, clinical and radiological improvement accompanied that of biological values, without any side-effects.

Systemic glucocorticoids are the treatment of choice for acute ABPA and exacerbations of ABPA. The Cystic Fibrosis Foundation Consensus Conference on ABPA did not propose a specific treatment plan [1]. Two small, uncontrolled clinical trials evaluated glucocorticoids in ABPA with different glucocorticoid regimens that varied in doses and durations (from 2 to 6 months) [2]. Short-term glucocorticoids reduced the number of exacerbations and improved lung function, but caused long-term sideeffects (diabetes, dyslipidaemia and osteopenia) and exposed patients to the risk of severe infection (ABPA progression to invasive pulmonary aspergillosis).

Systemic antifungal treatments have been recommended in association with glucocorticoids: the objective being the attenuation or even the eradication of the intrabronchial Aspergillus burden, in order to decrease or stop glucocorticoid therapy. Itraconazole is the antifungal agent of choice for this indication, according to the results of two randomised controlled trials 

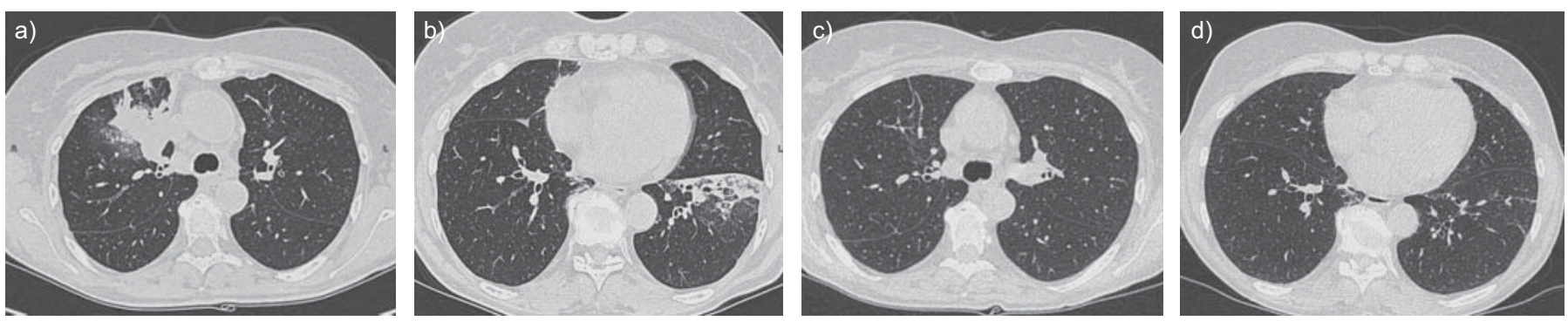

FIGURE 1. a, b) Computed tomography (CT) images at allergic bronchopulmonary aspergillosis deterioration. a) Section through the upper zones: note right apical consolidation and bronchocele associated with b) left basal consolidation and bronchiectasis. c, d) CT images 1 month after initiation of nebulised liposomal amphotericin B. Note complete resolution of consolidation (residual bronchiectasis) on sections through the c) upper and d) lower zones.

showing the superiority of the antifungal arm [2]. Itraconazole doses varied from 200 to $400 \mathrm{mg}^{-\mathrm{day}^{-1}}$ and follow-up did not extend beyond the 16-week treatment period. Furthermore, itraconazole use also raises several issues, such as drug interactions and hepatic toxicity [3]. In our case, the absence of relapse 12 months after steroid discontinuation could indicate that the addition of nebulised LAmB to oral glucocorticoids would be more effective for controlling ABPA exacerbations than steroids alone, as shown with itraconazole. Indeed, the addition of itraconazole to oral glucocorticoids decreases the number of ABPA exacerbations compared with glucocorticoids alone (mean \pm SEM $0.93 \pm 0.4$ versus $2.4 \pm 0.2$ exacerbations per patient and per year, respectively) [4].

The first stage in the pathogenesis of ABPA includes the inhalation of Aspergillus spp. spores, the colonisation of the airways by germinated Aspergillus and its proliferation in airway mucus. The administration of an antifungal treatment by inhalation could provide a new, simple therapeutic approach consistent with the pathophysiology of ABPA. Nebulised LAmB has been proposed to prevent invasive fungal infections in heart and/or lung transplant patients, and to cure patients refractory to conventional treatment [5]. A randomised, placebo-controlled trial performed in neutropenic patients demonstrated a dramatic reduction of invasive aspergillosis with $12.5 \mathrm{mg}$ LAmB administered twice weekly (14\% and $4 \%$ in the placebo and LAmB groups, respectively) [6]. In a cohort study of 27 lung transplant patients, a single weekly administration of nebulised LAmB ( $25 \mathrm{mg}$ ) yielded levels of amphotericin B in patient bronchoalveolar lavage that were high enough to inhibit growth of Aspergillus spp. $\left(>2 \mu \mathrm{g} \cdot \mathrm{mL}^{-1}\right)$, which persisted for 2 weeks [7]. Furthermore, nebulised LAmB tolerance has been reported as being quite satisfactory, particularly since the use of lipid formulations, which contain phospholipids and cholesterol that have properties similar to those of the natural surfactant [8]. Nebulised LAmB also decreases the risk of drug interactions observed with azoles. It allows higher lung tissue concentrations (compared with systemic administration) and only limited passage into blood plasma, as demonstrated by almost undetectable serum concentrations after administration of nebulised LAmB [7]. Finally, data regarding the efficacy of nebulised amphotericin B in ABPA are based on a few case reports; these suggest biological and clinical efficacy along with the possibility of reducing oral prednisolone doses, although they did not consider liposomal formulations $[8,9]$.
Yet another problem is the emergence of azole resistance and the use of a fungicide, such as nebulised AmBisome ${ }^{\circledR}$ (Astellas Pharma US Inc., Deerfield, IL, USA), could be of interest in limiting the risks of emerging azole-resistant strains and subsequent failure to respond to therapy [10].

In conclusion, this particular case demonstrates the efficacy of nebulised LAmB in controlling prednisolone-dependent ABPA. No relapse occurred 12 months after discontinuation of glucocorticoid therapy when nebulised LAmB had been continued as maintenance therapy for 6 months. In our opinion, in an attempt to reduce recourse to systemic glucocorticoid therapy and because the emergence of azole-resistance of Aspergillus is becoming a major concern, nebulised LAmB may have a place in the treatment of acute exacerbations of ABPA and, possibly, in the prevention of ABPA exacerbations. However, the efficacy and the safety of this treatment should be further investigated in a phase II prospective trial.

Cendrine Godet*, Jean-Claude Meurice", France Roblot*, Catherine Kauffmann-Lacroix", Marion Verdaguer", JeanPierre Frat ${ }^{+}$and Jacques Cadranel ${ }^{\S}$

*Service des Maladies Infectieuses, "Service de Pneumologie, 'Service de Parasitologie et Mycologie, ${ }^{+}$Service de Réanimation Médicale, Hôpital la Milétrie, Poitiers, and ${ }^{\S}$ Service de Pneumologie et Réanimation, AP-HP, Hôpital Tenon, Paris, France.

Correspondence: C. Godet, Service de Maladies Infectieuses et Tropicales, CHRU Poitiers, 2 rue de la Milétrie, 86021 Poitiers cedex, France. E-mail: c.godet@chu-poitiers.fr

Statement of Interest: Statements of interest for C. Godet and J. Cadranel can be found at www.erj.ersjournals.com/site/misc/ statements.xhtml

\section{REFERENCES}

1 Stevens DA, Moss RB, Kurup VP, et al. Allergic bronchopulmonary aspergillosis in cystic fibrosis-state of the art: Cystic Fibrosis Foundation Consensus Conference. Clin Infect Dis 2003; 37: 225-264.

2 Walsh TJ, Anaissie EJ, Denning DW, et al. Treatment of aspergillosis: clinical practice guidelines of the Infectious Diseases Society of America. Clin Infect Dis 2008; 46: 327-360. 
3 Leon EE, Craig TJ. Antifungals in the treatment of allergic bronchopulmonary aspergillosis. Ann Allergy Asthma Immunol 1999; 82: 511-516.

4 Salez F, Brichet A, Desurmont S, et al. Effect of itraconazole therapy in allergic bronchopulmonary aspergillosis. Chest 1999; 116: $1665-1668$.

5 Montoya JG, Chaparro SV, Celis D, et al. Invasive aspergillosis in the setting of cardiac transplantation. Clin Infect Dis 2003; 37: 281-292.

6 Rijnders BJ, Cornelissen JJ, Slobbe L, et al. Aerosolized liposomal amphotericin B for the prevention of invasive pulmonary aspergillosis during prolonged neutropenia: a randomized, placebo-controlled trial. Clin Infect Dis 2008; 46: 1401-1408.

7 Monforte V, Ussetti P, López R, et al. Nebulized liposomal amphotericin B prophylaxis for Aspergillus infection in lung transplantation: pharmacokinetics and safety. J Heart Lung Transplant 2009; 28: 170-175.

8 Laoudi Y, Paolini JB, Grimfeld A, et al. Nebulised corticosteroid and amphotericin B: an alternative treatment for ABPA? Eur Respir J 2008; 32: 908-909.

9 Proesmans M, Vermeulen F, Vreys M, et al. Use of nebulized amphotericin B in the treatment of allergic bronchopulmonary aspergillosis in cystic fibrosis. Int J Pediatr 2010; 23: 376287.

10 Howard SJ, Cerar D, Anderson MJ, et al. Frequency and evolution of Azole resistance in Aspergillus fumigatus associated with treatment failure. Emerg Infect Dis 2009; 15: 1068-1076.

\section{Proficiency testing of first- and second-line anti- tuberculosis drugs in Italy}

\section{To the Editors:}

The emergence of drug-resistant tuberculosis (TB) is an increasing threat to public health in industrialised countries; thus, it is important to supervise mycobacteriology laboratories by performing periodic proficiency of anti-TB drug susceptibility testing (DST). In 1994, the World Health Organization (WHO) and the International Union against Tuberculosis and Lung Diseases developed a global project of anti-TB drug resistance surveillance to assist countries via a network of supranational reference laboratories (SRLs). Proficiency test (PT) results of firstline anti-TB drugs have been reported for the SRL network [1] and for some individual countries $[2,3]$.

The SRL in Rome, Italy, coordinated two PTs of first-line drugs in endemic countries in 2002-2006 [4] and two PTs of first-line drugs in Italy in 1998-2000 [5, 6]. The present study aims to verify whether the quality of DST in Italy changed after that time; to this end, a comprehensive survey of five PTs during a 13-yr period (1998-2010) is reported here, together with a pilot round of second-line drug PTs in 2010.

Laboratories covering 18 out of 20 Italian regions participated in the PT exercise: 22 laboratories in 1998, 20 in 2000, 28 in 2003, 29 in 2007 and 30 in 2010. To maintain knowledge and skills, the laboratories were selected by the SRL on the basis of the number of patient samples analysed for DST. For instance, a mean of 88 first-line DSTs per laboratory (range 21-357) were performed in 2009. In 2010, 13 laboratories with a mean of 113 first-line and six second-line DSTs per laboratory in 2009 also performed the second-line drug PT. A mean of nine second-line DSTs per laboratory was performed in 2010. The Mycobacterium tuberculosis panels for first- and second-line drug PTs distributed by the Rome SRL to the mycobacteriology laboratories (all from public hospitals) contained only WHO-characterised strains received annually from the WHO coordinating centres in Ottawa, Canada (4th and 5th rounds [7]) and Antwerp, Belgium (8th, 10th and 14th rounds [1]). Panel strains were selected with the aim of achieving a $50 \%$ representation of resistance to streptomycin $(\mathrm{SM})$, isoniazid $(\mathrm{INH})$, rifampicin (RMP), ethambutol (EMB), kanamycin (KM), amikacin (AK), capreomycin (CP) and ofloxacin (OFL) in various combinations [1]. In 1998-2007, 20 strains (10 pairs) were sent to the laboratories for first-line PTs. The pattern of resistance/susceptibility to $\mathrm{SM}, \mathrm{INH}, \mathrm{RMP}$ and EMB, respectively, was: 10/10,14/6, 10/10 and 6/14 in 1998; 8/12, 16/ $4,10 / 10$ and $12 / 8$ in $2000 ; 8 / 12,12 / 8,6 / 14$ and $10 / 10$ in 2003; and $8 / 12,10 / 10,6 / 14$ and $8 / 12$ in 2007. In 2010, 10 strains (five pairs) of the 14th $\mathrm{WHO}$ round were sent to the laboratories for both first- and second-line PTs. The pattern of resistance/ susceptibility to SM, INH, RMP, EMB, KM, AK, CP and OFL was $6 / 4,4 / 6,6 / 4,2 / 8,6 / 4,2 / 8,4 / 6$ and $2 / 8$, respectively. The use of 10 strains for second-line PT was adequate to assess the quality of the laboratories, which analysed a mean of nine patient samples for second-line DST in 2010 (see earlier). Each laboratory used their routine DST method to test the samples they received. Procedures for first-line drugs included testing on solid media (proportion method in Löwenstein-Jensen (LJ) medium) and liquid media (BACTEC 460TB system and Manual MGIT or MGIT 960 system (Becton Dickinson, Sparks, MD, USA), or MB/ BacT (MB) (Organon Teknika, Boxtel, the Netherlands)). For the first-line drug PTs, the use of LJ and BACTEC decreased from $43.5 \%$ and $39.1 \%$ in 1998 to $0 \%$ and $0 \%$ in 2010, respectively. Conversely, the use of MGIT increased from $17.4 \%$ in 1998 to $100 \%$ in 2010. Only one laboratory used MB in 2000. Second-line drug PTs were performed with the MGIT system or in LJ medium using $5,1,2.5$ or $2 \mu \mathrm{g} \cdot \mathrm{mL}^{-1}$, or $30,40,40 \mathrm{or} 2 \mu \mathrm{g} \cdot \mathrm{mL}^{-1}$ of $\mathrm{KM}, \mathrm{AK}, \mathrm{CP}$ or OFL, respectively $[8,9]$. Sensitivity testing for one first-line drug (pyrazinamide) and for some second-line drugs (e.g. cycloserine) are not reliable and, therefore, were not compared in a PT programme. Results were reported as "resistant", "susceptible" or "no result due to lack of strain growth". Data were evaluated for sensitivity (ability to detect true resistance), specificity (ability to detect true susceptibility) and efficiency (ratio between the number of correct results and the total number of results), according to the WHO format [1, 7]. In each round, the laboratories that obtained $<90 \%$ efficiency retested the same strain panel for that/those drug(s); laboratories were informed of the total numbers of discrepancies for one or more drugs, but not about individual strain discrepancies [4]. 